\title{
A Binarization Algorithm Based on Shade-Planes for Road Marking Recognition
}

\author{
Tomohisa Suzuki $^{1}$, Naoaki Kodaira ${ }^{1}$, Hiroyuki Mizutani ${ }^{1}$, Hiroaki Nakai $^{2}$, \\ and Yasuo Shinohara ${ }^{2}$ \\ 1 Toshiba Solutions Corporation \\ 2 Toshiba Corporation
}

\begin{abstract}
A binarization algorithm tolerant to both gradual change of intensity caused by shade and the discontinuous changes caused by shadows is described in this paper. This algorithm is based on "shadeplanes", in which intensity changes gradually and no edges are included. These shade-planes are produced by selecting a "principal-intensity" in each small block by a quasi-optimization algorithm. One shade-plane is then selected as the background to eliminate the gradual change in the input image. Consequently, the image, with its gradual change removed, is binarized by a conventional global thresholding algorithm. The binarized image is provided to a road marking recognition system, for which influence of shade and shadows is inevitable in the sunlight.
\end{abstract}

\section{Introduction}

The recent evolution of car electronics such as low power microprocessors and in-vehicle cameras has enabled us to develop various kinds of on-board computer vision systems [1] 2]. A road marking recognition system is one of such systems. GPS navigation devices can be aided by the road marking recognition system to improve their positioning accuracy. It is also possible to give the driver some advice and cautions according to the road markings.

However, influence of shade and shadows, inevitable in the sunlight, is problematic to such a recognition system in general. The road marking recognition system described in this paper is built with a binarization algorithm that performs well even if the input image is affected by uneven illumination caused by shade and shadows.

To cope with the uneven illumination, several dynamic thresholding techniques were proposed. Niblack proposed a binarization algorithm, in which a dynamic threshold $t(x, y)$ is determined by the mean value $m(x, y)$ and the standard-deviation $\sigma(x, y)$ of pixel values in the neighborhood as follows [4].

$$
t(x, y)=m(x, y)+k \sigma(x, y)
$$

where $(x, y)$ is the coordinate of the pixel to be binarized, and $k$ is a predetermined constant. This algorithm is based on the assumption that some of the neighboring pixels belong to the foreground. The word "Foreground" means 
characters printed on a paper, for example. However, this assumption does not hold in the case of a road surface where spaces are wider than the neighborhood.

To determine appropriate thresholds in such spaces, some binarization algorithms were proposed [5] 6]. In those algorithms, an adaptive threshold surface is determined by the pixels on the edges extracted from the image. Although those algorithms are tolerant to the gradual change of illumination on the road surface, edges irrelevant to the road markings still confound those algorithms.

One of the approaches for solving this problem is to remove the shadows from the image prior to the binarization. In several preceding researches, this shadow removal was realized by using color information. It was assumed in those methods that changes of color are seen on material edges [7] [8]. Despite fair performance for natural sceneries in which various colors tend to be seen, those algorithms does not perform well if the brightness is solely different and no different colors are seen.

Since many road markings tend to appear almost monochrome, we have concluded that the binarization algorithm for the road marking recognition has to tolerate influence of shade and shadows without depending on color information. To fulfill this requirement, we propose a binarization algorithm based on shade-planes. These planes are smooth maps of intensities, and these maps do not have edges which may appear, for an example, on material edges of the road surface or on borders between shadows and sunlit regions. In this method, the gradual change of intensity caused by shade is isolated from the discontinuous change of intensity. An estimated map of background intensity is found in these shade-planes. The input image is then modified to eliminate the gradual change of intensity using the estimated background intensity. Consequently, a commonly used global thresholding algorithm is applied to the modified image.

This binarized image is processed by segmentation, feature extraction and classification which are based on algorithms employed in conventional OCR systems. These conventional algorithms become feasible due to reduction of artifacts caused by shade and shadows with the proposed binarization algorithm.

The recognition result by this system is usable in various applications including GPS navigation devices. For instance, the navigation device can verify whether the vehicle is travelling in the appropriate lane.

In the case shown in Fig[1, the car is travelling in the left lane, in which all vehicles must travel straight through the intersection, despite the correct route heading right. The navigation device detects this contradiction by verifying the road markings which indicate the direction the car is heading for, so that it can suggest the driver to move to the right lane in this case.

It is also possible to calibrate coordinates of the vehicle gained by a GPS navigation device using other coordinates which are calculated from relative position of a recognized road marking and its position on the map.

As a similar example, Ohta et al. 3] proposed a road marking recognition algorithm to give drivers some warnings and advisories. Additionally, Charbonnier et al. 2] developed a system that recognizes road markings and repaints them. 


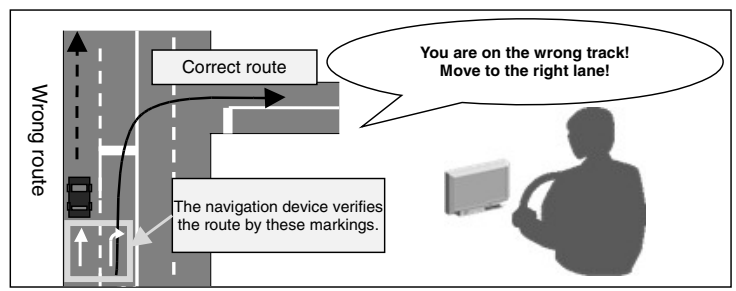

Fig. 1. Lane change suggested by verifying road markings

This paper is organized as follows. The outline of the proposed recognition system is described in Sect 2. Influence of shade and shadows on the images taken by the camera and the binarization result is described in Sect 3 . The proposed binarization algorithm is explained in Sect 4 . The experimental result of the binarization and the recognition system are shown in Sect 5 , and finally, we summarize with some conclusions in Sect 6 .

\section{Outline of Overall Road Marking Recognition System}

The recognition procedure in the proposed system is performed by the following steps: perspective transformation [9], binarization which is the main objective in this paper, lane detection, segmentation, pattern matching and post processing.

As shown in Fig 2, the camera is placed on the rear of the car and directed obliquely to the ground as shown in Fig[3]Since the image taken by a camera in an oblique angle is distorted perspectively, perspective transformation is performed for the image as seen in Fig, to produce an image without distortion.

The transformed image is then binarized by the proposed algorithm to extract the patterns of the markings. (See Fig 5) We describe the detail of this algorithm later in Sect 4

The next step is to extract the lines drawn along the lane on the both sides, in which the road markings are to be recognized. These lines are detected by edges along the road as in the system previously proposed [10].

The road markings, which are shown in Fig [6 are recognized by this system. The segmentation of these symbols is performed by locating their bounding rectangles. Each edge of the bounding rectangles is determined by the horizontal and vertical projection of foreground pixels between the lines detected above.

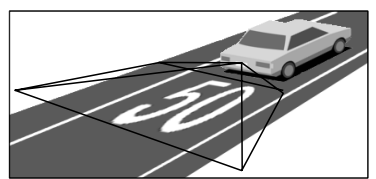

Fig. 2. Angle of the camera

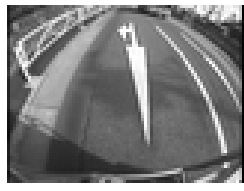

Fig. 3. Image taken by the camera 


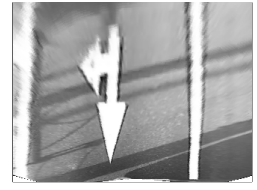

Fig. 4. Image processed by perspective transform

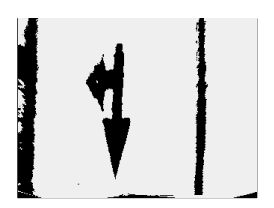

Fig. 5. Binarized image

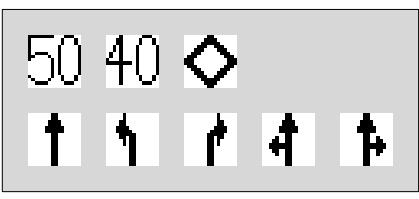

Fig. 6. Recognized road markings

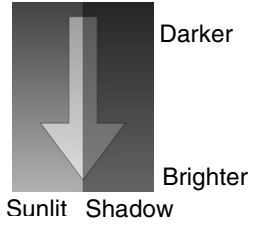

Fig. 7. Road marking with shade and a shadow

The segmented symbols are then recognized by the subspace method [11]. The recognition results are corrected by following post-processes:

- The recognition result for each movie frame is replaced by the most frequently detected marking in neighboring frames. This is done to reduce accidental misclassification of the symbol.

- Some parameters (size, similarity and other measurements) are checked to prevent false detections.

- Consistent results in successive frames are aggregated to one marking.

\section{The Influence of Shade, Shadows and Markings on Images}

In the example shown in Fig, 4 , we can see the tendency that the upper right part of the image is brighter than the lower left corner. In addition, the areas covered by the shadows casted by objects beside the road are darker than the rest. As seen in this example, the binarization algorithm applied to this image is to be tolerant to both the gradual changes of intensity caused by shade and the discontinuous change of intensity caused by shadows on the road surface.

For example, these changes of intensity are illustrated in Fig 7 In this example, the gradual change of intensity caused by shade is seen along the arrow, and the discontinuous change of intensity caused by shadow is seen perpendicular to the arrow. From these changes, the discontinuous change on edges of the road marking, the outline of the arrow in this case, has to be used to binarize the image without influence of shade and shadows. 


\section{The Proposed Binarization Algorithm}

In this section, the proposed binarization algorithm is presented.

\subsection{Pre-processing Based on the Background Map}

In the proposed algorithm, the gradual change of intensity in the input image is eliminated from the input image prior to the binarization by a global thresholding method - Otsu's method [12]. This pre-processing is illustrated by Fig 8 and is performed by producing a modified image (Fig $8(\mathrm{c})$ ) from the input image $(\operatorname{Fig}[8(a))$ and a map of background intensity $(\operatorname{Fig}[(b))$ with the following equation. This pre-processing flattens the background to make a global thresholding method applicable.

$$
g(x, y)=\frac{f(x, y)}{l(x, y)}
$$

In this pre-processing, a map of the background intensity called "background map" is estimated by the method described in the following section.

\subsection{Estimation of a Background Map by Shade-Planes}

In this section, the method for estimating a background map is described.

\subsubsection{Detection of Principal-Intensities}

An intensity histogram calculated in a small block shown as "small block" in Fig 9 usually consists of peaks at several intensities corresponding to the regions marked with symbols A-D in this figure. We call these intensities "principalintensity".

The input image is partitioned into small blocks as a $\mathrm{PxQ}$ matrix in this algorithm, and the principal-intensities are detected in these blocks. Fig 10 is an example of detected principal-intensities. In this figure, the image is divided into $8 \times 8$ blocks. Each block is divided into sub-blocks painted by a principalintensity. The area of each sub-block indicates the number of the pixels that have the same intensity in the block. As a result, each of the detected principalintensities corresponds to a white marking, grey road surface or black shadows.

In each block, one of the principal-intensities is expected to be the intensity in the background map at the same position. The principal-intensity corresponding to the background is required to be included in most of the blocks in the proposed

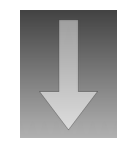

(a) Input image $f(x, y)$

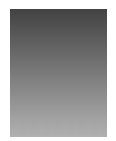

(b) Background map $l(x, y)$

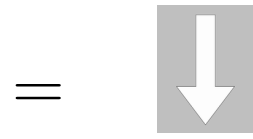

(c) Modified image $g(x, y)$

Fig. 8. A pre-processing is applied to input image 


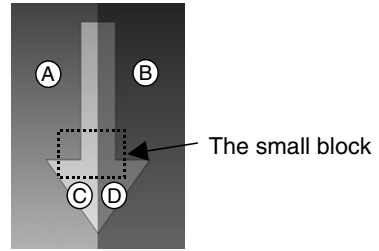

(a) Block in which the histogram is computed

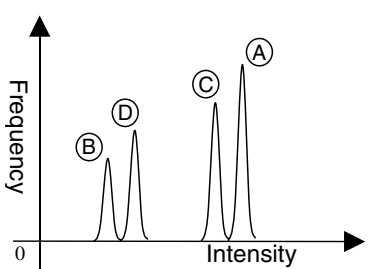

(b) Intensity histogram

Fig. 9. Peaks in a histogram for a small block

method. Though, gray sub-blocks corresponding to the background are missing in some blocks at the lower-right corner of the Fig 10 .

To compensate the absence of principal-intensities, the histogram averaged in the $5 \times 3$ neighbor blocks are calculated instead. Fig 11 shows the result by this modified scheme. As a result, the grey sub-blocks can be observed in all blocks.

\subsubsection{The Shade-Planes}

In this method, the maps of principal-intensities are called "shade-plane", and a bundle of the plural shade-planes is called a "shade-plane group". Each shadeplane is produced by selecting the principal-intensities for each block as shown in Fig 12. In this example, black sub-blocks among the detected principal-intensities correspond to the road surface in shadows, the grey sub-blocks correspond to the sunlit road surface and the white sub-blocks correspond to markings. The principalintensities corresponding to the sunlit road surface are selected in the shade-plane \#1 and those corresponding to road marking are selected in shade-plane \#2.

Principal-intensities in each shade-plane are selected to minimize the following criterion $E$. This criterion is designed, so that the shade-plane represents gradual change of intensities.

$$
E=\sum_{s=1}^{Q} \sum_{r=1}^{P-1}\{L(r+1, s)-L(r, s)\}^{2}+\sum_{s=1}^{Q-1} \sum_{r=1}^{P}\{L(r, s+1)-L(r, s)\}^{2}
$$

where $L(r, s)$ stands for the principal-intensity selected in the block $(r, s)$.

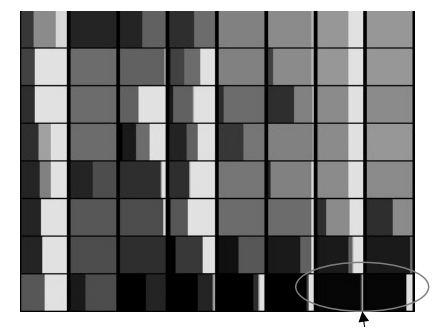

Gray sub-blocks are missing here

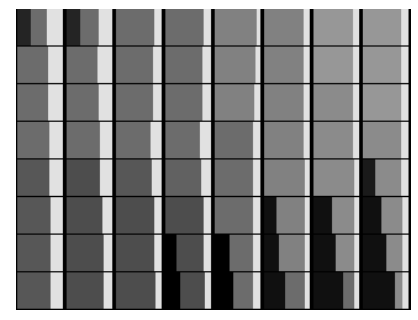

Fig. 11. Results with averaged histograms 


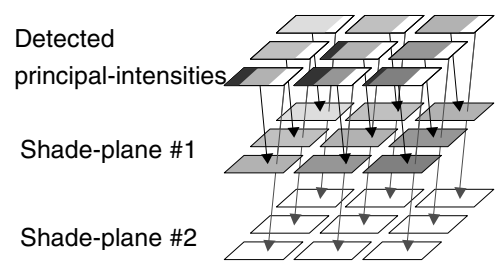

Fig. 12. Shade-planes

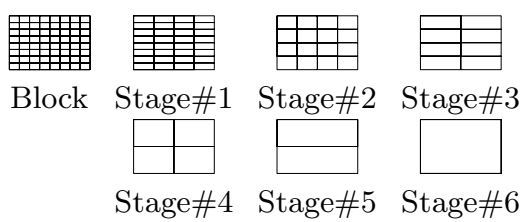

Fig. 13. Merger of areas

The number of the possible combinations of the detected principal-intensities is extremely large. Therefore, a quasi-optimization algorithm with the criterion $E$ is introduced to resolve this problem.

During the optimization process, miniature versions of a shade-plane called a "sub-plane" are created. The sub-planes in the same location form a group called "sub-plane group". The sub-plane groups cover the whole image without overlap altogether. Pairs of adjoining sub-plane groups are merged to larger sub-plane groups step by step, and they finally form the shade-plane group, which is as large as the image. Each step of this process is expressed by "Stage\#n" in the following explanation.

Fig 13 shows the merging process of sub-plane groups in these stages. "Blocks" in the Fig 13 indicates the matrix of blocks, and "Stage\#n" indicates the matrix of sub-plane groups in each stage. In the stage\#1, each pair of horizontally adjoining blocks is merged to form a sub-plane group. In the stage\#2, each pair of vertically adjoining sub-plane groups is merged to form a larger subplane group. This process is repeated recursively in the same manner. Finally, "Stage\#6" shows the shade-plane group.

The creation process of a sub-plane group in stage\#1 is shown in Fig.14. In this figure, pairs of principal-intensities from a pair of blocks are combined to create candidates of sub-planes. Consequently, the criterion $E$ is evaluated for each created candidate, and a new sub-plane group is formed by selecting the two sub-planes with the least value of criterion $E$.

For the stage\#2, Fig 15] shows creation of a larger sub-plane group from a pair of sub-plane groups previously created in stage\#1. Contrarily to the stage\#1, the candidates of the new sub-plane group are created from sub-plane groups instead of principal-intensities.

\subsubsection{Selection of the Shade-Planes}

A shade plane is selected from the shade-plane group produced by the algorithm described in Sect 4.2.2 as the background map $l(r, s)$. This selection is performed by the following procedure.

1. Eliminate shade-planes similar to another if a pair of shade-planes shares half or more of the principal-intensities.

2. Sort the shade-planes in descending order of the intensity. 


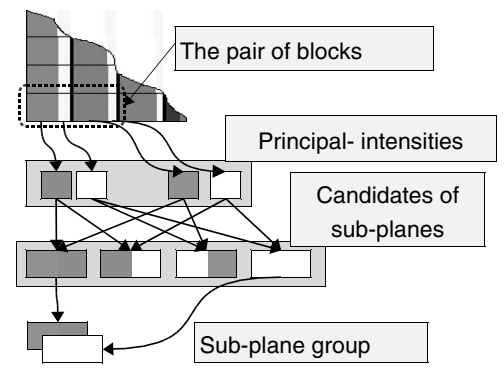

Fig. 14. Sub-planes created in stage\#1

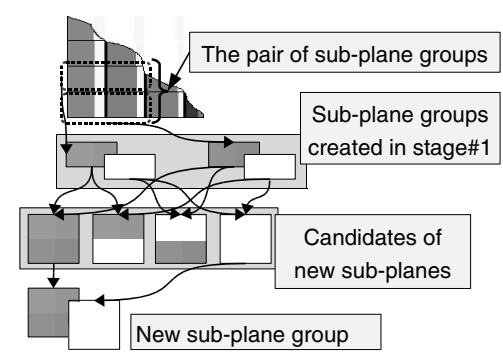

Fig. 15. Sub-planes created in stage \#2

3. Select the shade-plane that is closest to the average of shade-planes produced in the preceding $K$ frames. The similarity of shade-planes is computed as Euclidian distance.

\section{The Experimental Results}

Fig 16 and Fig 17 show the results of the proposed binarization algorithm. In each of the figures, the image (a) is the input, the image (b) is the background map, and the image (c) is the binarization result. As a comparison, the result by Niblack's method is shown in the image (d). Additionally, the image (e) shows the shade-planes produced by the proposed algorithm.

In Fig[16(e), change of intensity corresponding to the marking is seen in "Plane\#1" and change of intensity corresponding to road surface is seen in "Plane\#2". "Plane\#3" and "Plane\#4" are useless in this case. These changes of intensity corresponding to the marking and road surface are also seen in Fig[17(e) in "Plane\#2" and "Plane\#1" respectively.

Contrarily, in the Fig[16(d) and Fig[17(d), the conventional method [4] did not work well.

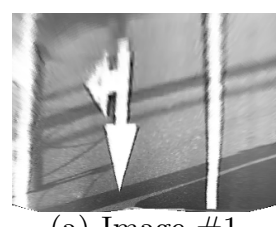

(a) Image \#1

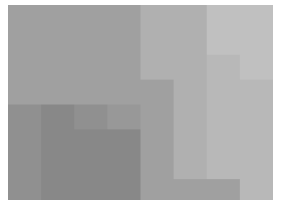

(b) Background

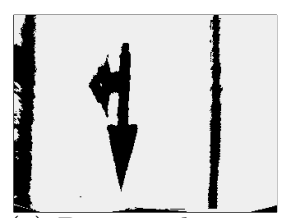

(c) Binarized image

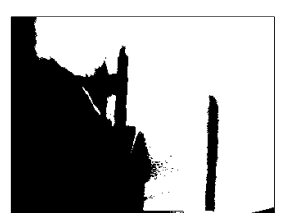

(d) Niblack's method

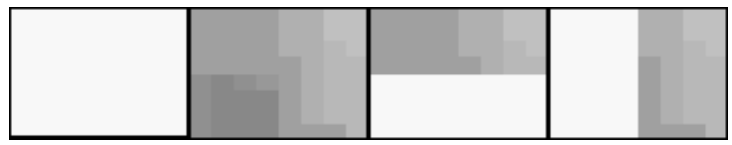

(e) The shade-planes produced by this algorithm

Fig. 16. Experimental results for the sample image\#1 


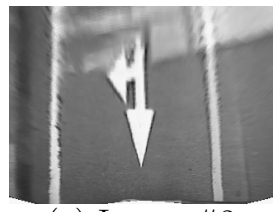

(a) Image \#2

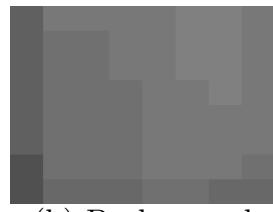

(b) Background

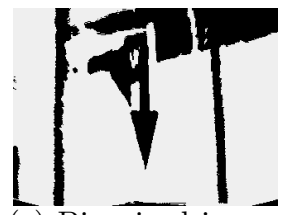

(c) Binarized image

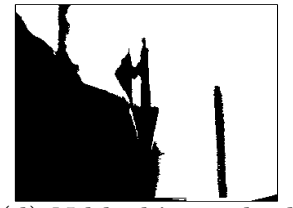

(d) Niblack's method

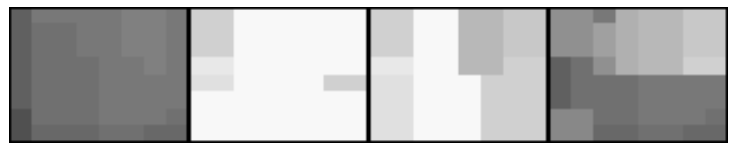

(e) The shade-planes produced by this algorithm

Fig. 17. Experimental results for the sample image\#2

Table 1. Recognition performance

\begin{tabular}{ccccccc}
\hline Movie No. Frames & Markings & $\begin{array}{c}\text { Detected } \\
\text { markings }\end{array}$ & Errors Precision & $\begin{array}{c}\text { Recall } \\
\text { rate }\end{array}$ \\
\hline 1 & 27032 & 64 & 53 & 0 & $100 \%$ & $83 \%$ \\
2 & 29898 & 131 & 110 & 0 & $100 \%$ & $84 \%$ \\
3 & 63941 & 84 & 65 & 0 & $100 \%$ & $77 \%$ \\
\hline total & 120871 & 279 & 228 & 0 & $100 \%$ & $82 \%$ \\
\hline
\end{tabular}

The binarization error observed in the upper part in Fig 17)(c) is caused by selecting "Plane\#1", which corresponds to the shadow region that covers the most area in the image. This led to the binarization error in the sunlit region, for which "Plane\#4" would be better.

We implemented the road marking recognition system with the proposed binarization algorithm on a PC with $800 \mathrm{MHz}$ P3 processor as an experimental system. The recognition system described above was tested with the QVGA movies taken on the street. The processing time per frame was $20 \mathrm{msec}$ on average, and was fast enough to process movie sequences by 30fps. Table 1 shows the recognition performance of these movies in this experiment. The average recall rate of marking-recognition was $82 \%$ and no false positives were observed throughout 120,871 frames.

\section{Conclusion}

A binarization algorithm that tolerates both shade and shadows without color information is described in this paper. In this algorithm, shade-planes associated to gradual changes of intensity are introduced. The shade-planes are produced by a quasi-optimization algorithm based on the divide and conquer approach. Consequently, one of the shade-planes is selected as an estimated background 
to eliminate the shade and enable conventional global thresholding methods to be used. In the experiment, the proposed binarization algorithm has performed well with a road marking recognition system.

An input image almost covered by a shadow showed an erroneous binarization result in a sunlit region. We are now seeking for an enhancement to mend this problem.

\section{References}

1. Bertozzi, M., Broggi, A., Cellario, M., Fascioli, A., Lombardi, P., Porta, M.: Artificial Vision in Road Vehicles. Proc. IEEE 90(7), 1258-1271 (2002)

2. Charbonnier, P., Diebolt, F., Guillard, Y., Peyret, F.: Road markings recognition using image processing. In: IEEE Conference on Intelligent Transportation System (ITSC 1997), November 9-12, 1997, pp. 912-917 (1997)

3. Ohta, H., Shiono, M.: An Experiment on Extraction and Recognition of Road Markings from a Road Scene Image, Technical Report of IEICE, PRU95-188, 199512 , pp. 79-86 (in Japanese)

4. Niblack: An Introduction to Digital Image Processing, pp. 115-116. Prentice-Hall, Englewood Cliffs (1986)

5. Yanowitz, S.D., Bruckstein, A.M.: A new method for image segmentation. Comput.Vision Graphics Image Process. 46, 82-95 (1989)

6. Blayvas, I., Bruckstein, A., Kimmel, R.: Efficient computation of adaptive threshold surfaces for image binarization. In: Proceedings of the 2001 IEEE Computer Society Conference on Computer Vision and Pattern Recognition, December 2001, vol. 1, pp. $737-742(2001)$

7. Finlayson, G.D., Hordley, S.D., Cheng Lu Drew, M.S.: On the removal of shadows from images. IEEE Transactions on Pattern Analysis and Machine Intelligence 28, 59-68 (2006)

8. Nielsen, M., Madsen, C.B.: Graph Cut Based Segmentation of Soft Shadows for Seamless Removal and Augmentation. In: Ersbøll, B.K., Pedersen, K.S. (eds.) SCIA 2007. LNCS, vol. 4522, pp. 918-927. Springer, Heidelberg (2007)

9. Forsyth, D.A., Ponce, J.: Computer Vision A Modern Approach, pp. 20-37. Prentice Hall, Englewood Cliffs (2003)

10. Nakayama, H., et al.: White line detection by tracking candidates on a reverse projection image, Technical report of IEICE, PRMU 2001-87, pp. 15-22 (2001) (in Japanese)

11. Oja, E.: Subspace Methods of Pattern Recognition. Research Studies Press Ltd. (1983)

12. Otsu, N.: A threshold selection method from gray-level histograms. IEEE Trans. Sys. Man Cyber. 9(1), 62-66 (1979) 